Voix et Images

volxetimages

\title{
Métaphore et métonymie dans Menaud, maître-draveur
}

\section{Anthony Purdy}

Volume 12, numéro 1 (34), automne 1986

Québec-Amérique latine

URI : https://id.erudit.org/iderudit/200607ar

DOI : https://doi.org/10.7202/200607ar

Aller au sommaire du numéro

Éditeur(s)

Université du Québec à Montréal

ISSN

0318-9201 (imprimé)

1705-933X (numérique)

Découvrir la revue

Citer cet article

Purdy, A. (1986). Métaphore et métonymie dans Menaud, maître-draveur. Voix et Images, 12(1), 68-85. https://doi.org/10.7202/200607ar d'utilisation que vous pouvez consulter en ligne.

https://apropos.erudit.org/fr/usagers/politique-dutilisation/ 


\title{
Métaphore et métonymie dans Menaud, maûtre-draveur
}

\author{
par Anthony Purdy, Université de l'Alberta
}

- Ce matin, il lui semble que tout le passé est là, dans ce cirque remué de pourpre et d'or.

Le décor a provoqué!

(Menaud, p. 152)

À parcourir la littérature critique sur Menaud, maître-draveur, on est frappé par un malaise presque universel devant ce texte excessif, démesuré. Le plus souvent, ce malaise est attribuable à une certaine hésitation en ce qui concerne la détermination du genre littéraire auquel le livre appartiendrait. De Maurice Hébert, écrivant en 1937 - En résumé, Menaud est bien plus un récit poétique ou poétisé qu'un roman' - à Madeleine Ducrocq-Poirier en 1978 - L'exploitation poétique du récit en altère la substance romanesque; elle s'avère en être l'adversaire ${ }^{2}$-, la conclusion reste la même: que l'interférence du poétique et du romanesque dans ce livre se produisait au détriment du roman ${ }^{3}$. Le jugement porté par Réjean Robidoux et André Renaud est à cet égard typique: L'emploi systématique de la métaphore, le recours régulier à la manière épique ainsi que la fréquence des mouvements lyriques contribuent à faire de Menaud, maître-draveur une fresque héroïque où se transforme en allégorie l'aventure réaliste des personnages ${ }^{4}$. Parfois, le problème du genre se voit compliqué - mais aussi éclairé - par la question des différentes éditions de l'œuvre:

Il y a, dans la version de 1937, une profusion d'images, de métaphores qui se précipitent nerveusement, s'entrelacent, se croisent, et qui enlèvent à la prose l'allure romanesque... Plus brève en général et plus souple, la phrase de la deuxième version réalise cette exigence de l'art romanesque tout en rétablissant un équilibre quasi parfait entre la poésie, - dont le texte de 1944 reste imprégné, - et le roman comme genre littéraires.

C'est ainsi que François Ricard peut voir dans l'édition de 1944 la victoire d'un certain 'classicisme' sur l'ivresse romantique de 1937, victoire marquée par un retour au réalisme et une transition du lyrique au narratif ${ }^{6}$.

On aura remarqué le rôle prépondérant attribué par les critiques à la métaphore dans les rapports qui s'établissent entre les deux dimensions de l'ouvrage. C'est par la métaphore que, selon Ricard, le roman se détournerait du réel pour revêtir un aspect épique: 


\begin{abstract}
Par son ouverture sur la métaphore, l'écrivain établit autour de la simple aventure d'un draveur tout un réseau supérieur de relations qui lui confère les dimensions d'un travail herculéen. C'est en ce sens, surtout, que Menaud, maître-draveur peut être dit animé d'un souffle épique?
\end{abstract}

Mais, si la métaphore est le signe d'une transformation héroïque du réel, il faut constater que cette transformation reste entièrement subjective; si l'auteur de Menaud ne vise pas au naturalisme, c'est que sa vue est franchement intérieure, métaphorique; elle transpose au lieu de peindre, célèbre au lieu d'expliquer. Savard [...] s'introduit dans l'âme de son héros, c'est à travers la loupe de son émotion qu'il regarde les choses et les hommes, à travers le feu de sa passion qu'il les exprime ${ }^{8}$. La métaphore serait donc l'équivalent stylistique de la vision transformatrice, métaphysique, de Menaud lui-même, et, à la limite, de sa folie. Abordons donc notre sujet par une analyse «en petit» des images, pour en revenir par la suite à ces questions de grande envergure qui se posent sur le plan des structures thématiques et formelles de l'œuvre.

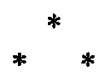

Confrontons tout de suite les deux phrases suivantes:
A. Et son esprit partit en course dans tous les sentiers de la forêt, multiple comme une meute sur des pistes de haut gibier ${ }^{9}$.
B. Il lui passait des idées folles qui lui tricolaient dans tous les chemins du cerveau comme des jeunesses qui reviennent des noces, en ripompette 10 .

Ce qui frappe quand on lit ces deux phrases ainsi juxtaposées, c'est la symétrie qui se manifeste à tous les niveaux entre les deux exemples. Tout d'abord, sur le plan du contenu, le sentiment évoqué de part et d'autre est essentiellement le même; il s'agit, dans les deux cas, d'une certaine excitation de la pensée: là c'est Menaud qui jouit de sa décision de partir à la défense de l'héritage, ici c'est Marie qui attend avec une impatience délicieuse la visite du Lucon. Ensuite, sur le plan de la forme, on constate la même structure rhétorique binaire (métaphore plus comparaison) avec, de $\mathbf{A}$ à $\mathbf{B}$, un léger déplacement syntaxique de l'élément qui sert de liaison entre les deux figures (multiple / en ripompette). Enfin, à l'intérieur même de la métaphore, on remarque une certaine isotopie sémantique (dans tous les sentiers de la forêt / dans tous les chemins du cerveau) qui vient renforcer la symétrie syntaxique.

Cependant, s'il existe, au niveau de l'écriture, des ressemblances saisissantes entre les deux phrases, il faut constater qu'il y a aussi des différences. En premier lieu, les deux comparaisons introduisent un contraste entre le thème de la chasse (comme une meute sur des pistes de haut gibier) et celui du mariage (comme des jeunesses qui reviennent des noces). De même, les 
deux métaphores évoquent l'opposition dialectique du dehors (sentiers de la forêt) et du dedans (chemins du cerveau), répétant ainsi, à l'intérieur même du jeu des images, l'une des grandes structures thématiques de l'œuvre. Enfin, sur le plan global, les deux phrases se présentent, respectivement, sous le signe du départ (partir en course) et sous celui du retour (revenir des noces).

Ce qui est intéressant ici, c'est que l'analyse différentielle, sur le plan des images, débouche nécessairement sur une lecture intégrale à un niveau supérieur: les différences qu'on a constatées sur le plan des phénomènes - chasse/noces, sentiers de la forêt/chemins du cerveau, départ/retour - sont toutes régies en fonction de certaines constantes structurales de l'univers romanesque: la dialectique du dehors et du dedans, l'opposition irréductible du masculin et du féminin, le contraste entre le nomade et le sédentaire, entre l'espace du départ - il s'était fait une fête de partir (p. 181) - et celui de l'attente, lui-même ambigu: la maison promise (p. 170)/ une cambuse funèbre (p. 181). C'est à ce titre que l'on peut parler, comme le fait Gérard Genette dans un essai sur Proust, de métaphores à fondement métonymique, ou métaphores diégétiques - diégétiques en ce sens que leur 'véhicule' est emprunté à la diégèse, c'est-à-dire à l'univers spatio-temporel du récit ". Or, il nous semble que l'analyse faite par Genette de ce genre de métaphore est exemplaire et pourrait nous fournir des aperçus précieux en ce qui concerne l'étude de l'imagerie savardienne. Essayons done de présenter les éléments les plus importants de son argument.

Le critique commence par une mise en garde: la métaphore, dit-il, est si importante chez Proust que l'on est tout naturellement porté, comme l'écrivain lui-même d'ailleurs, à en surestimer l'action au détriment d'autres relations sémantiques ${ }^{12}$. Parmi ces autres relations sémantiques, Genette s'attache tout particulièrement à ce qu'il appelle des transpositions typiquement métonymiques et dont il cite comme exemple les hypallages relevées par S. Ullmann dans son Style in the French Novel ${ }^{13}$. Dans le cas de Savard, on pourrait ranger dans la même catégorie une image telle que la grande paix dorée du soir (p. 19), image fondée, selon Ullmann, sur la contiguitté de deux sensations, sur leur coexistence dans le même contexte mental ${ }^{14}$. Cependant, à force de parcourir le texte de Menaud, on se voit obligé de reconnaître que, chez Savard comme chez Proust, les transpositions purement métonymiques restent assez rares. Mieux vaut donc suivre l'exemple de Genette, qui constate que faire sa part à la métonymie ne consistera pas à en dresser une liste concurrente en face de celle des métaphores, mais plutôt à montrer la présence et l'action des relations de 'coexistence' à l'intérieur même du rapport d'analogie : le rôle de la métonymie dans la métaphore ${ }^{15}$.

Pour étudier cette interpénétration de la métaphore et de la métonymie, Genette commence par confronter deux passages de la Recherche du temps perdu, le premier tiré du Côté de chez Swann et situé à Méséglise, le second se trouvant dans Sodome et Gomorrhe, lors du deuxième séjour à Balbec. Dans les deux cas, il s'agit d'une description d'un couple de clochers, manifestement fort semblables en leurs caractéristiques objectives essentielles: la 
forme aiguë ou effilée, la couleur jaune-roux, la surface rugueuse, écailleuse ou alvéolée ${ }^{16}$. Devant cette similitude apparente, le critique s'avoue perplexe: Sur ces données sensiblement identiques, pourquoi l'imagination du narrateur greffe-t-elle deux comparaisons toutes différentes, là entre les clochers et des épis, ici entre les (mêmes) clochers et des poissons ? ${ }^{17} \mathrm{Or}$, comme le dit $\mathrm{Ge}-$ nette, la raison en est assez évidente: dans les deux passages, c'est l'entourage, le contexte spatio-temporel, qui suggère la comparaison: Entre ces deux 'similitudes' virtuelles, Proust choisit en chaque occurrence celle qui s'adapte le mieux à la situation ou (c'est la même chose) au contexte: qualité terrienne de Méséglise, essence marine de Balbec ${ }^{18}$. En effet, il s'agit là d'un procédé tout à fait caractéristique de la démarche proustienne, procédé que l'on pourrait décrire en termes de la contrainte exercée sur le rapport métaphorique par des relations de contiguité.

Dans tous les exemples cités par Genette, c'est la proximité (spatiale, temporelle, psychologique) qui commande ou cautionne la ressemblance, ce qui donne lieu à une hypothèse générale pour le moins séduisante: dans tous ces exemples, la métaphore trouve son appui et sa motivation dans une métonymie: ainsi en va-t-il bien souvent chez Proust, comme si la justesse d'un rapprochement analogique, c'est-à-dire le degré de ressemblance entre les deux termes, lui importait moins que son authenticité, entendons par là sa fidélité aux relations de voisinage spatio-temporel ${ }^{19}$. Ce principe d'authenticité ou d'homogénéité. Genette le compare à la pratique de certains cuisiniers qui s'ingénient à assortir tel plat régional d'une sauce ou d'une garniture rigoureusement autochtone, et à l'accompagner d'un vin 'de pays', persuadés de la convenance, de l'harmonie gustative des produits d'un même terroir ${ }^{20}$.

Revenons à Savard. Dans les deux phrases que nous avons citées, le principe d'authenticité dont parle Genette est manifestement à l'œuvre. Dans les deux cas, la justesse du rapport analogique semble être subordonnée à sa fidélité au contexte diégétique, en l'occurrence avant tout psychologique; c'est ainsi que les idées de Menaud ressemblent à une meute partie en course dans la forêt à la poursuite de sa proie, tandis que celles de Marie prennent tout naturellement la forme de jeunesses qui reviennent des noces. Ici et là, on a affaire à un processus de motivation ou de naturalisation: les comparaisons paraissent "naturelles» dans le contexte du caractère des deux personnages. C'est en fonction de ceux-ci que les différences entre les deux figures sont conçues, toutes les oppositions thématiques que nous avons constatées découlant de cette opposition fondamentale entre père et fille.

La question se pose alors de savoir s'il s'agit là d'une constante de la démarche savardienne. L'authenticité des images serait-elle d'ordre avant tout psychologique, fondée sur le système des personnages? Comparons les deux phrases suivantes qui se rapprochent des exemples déjà cités en ce sens que l'on y voit les mêmes personnages - Menaud et Marie - et la même excitation de l'esprit:

C. Mais dès que Josime eut passé la porte, ce fut dans la tête à Menaud comme un torrent d'avril. 
D. Tout le long du jour, s'il en neigea des idées dans la tête de
Marie!

Deux images donc qui se ressemblent tant par leur contenu — c'est essentiellement le même état d'âme qui est évoqué dans les deux cas - que par le fait que leurs 'véhicules' - torrent d'avril/neige - sont tous les deux empruntés au monde naturel des saisons. Mais, à l'intérieur de cette symbolique des saisons, comment expliquer le choix de deux éléments très différents pour désigner, par analogie, la même chose? Qu'est-ce qui a commandé la sélection de l'élément torrent d'avril pour évoquer les idées de Menaud, tandis que celles de Marie ressembleraient plutôt à une tempête de neige? S'agirait-il tout simplement d'un indice de différenciation psychologique, comme dans le cas des exemples $\mathrm{A}$ et $\mathrm{B}$, ou y aurait-il d'autres facteurs qui entrent en jeu?

Pour répondre à ces questions, il faut rétablir le contexte diégétique immédiat des deux phrases. On constate alors que le choix du torrent d'avril est déterminé moins par l'opposition Menaud/Marie que par celle entre Menaud et son voisin Josime:

Josime vint faire son bout de veillée.

Il reprit son sujet de la veille: la récolte, les labours d'automne.

Menaud, pour la politesse, répondait oui, non, à ce qui lui barrait ainsi l'idée, à ces versets aussi lents que des chars chargés de gerbes.

Mais dès que Josime eut passé la porte, ce fut dans la lête à Menaud comme un torrent d'avril.

Le torrent d'avril évoque donc, par métonymie, le temps de la drave et s'oppose, non aux neiges de l'hiver, mais à la récolte, aux labours d'automne, aux chars chargés de gerbes, qui résument et justifient le personnage de Josime, le paysan sédentaire. Nous retrouvons ainsi, inscrite à l'intérieur même des images choisies, l'opposition thématique primordiale de l'œuvre, celle du nomade et du sédentaire, celle qui contraste, d'une part, la liberté du fier coureur de bois qui ne se laisse jamais enraciner au sol (p. 23) et, d'autre part, la petite vie, étroite, resserrée (p. 171) du défricheur. On rejoint par là l'opposition déjà esquissée entre l'espace du départ et l'espace de l'attente, Josime se trouvant, selon la logique de l'œuvre, du côté des femmes. Quant à la comparaison entre les idées de Marie et la neige, il s'agit là d'une simple contamination par proximité: la métaphore est suggérée et préparée par la neige qui tombe dehors et qui rend la campagne silencieuse et comme résignée (p. 165).

Dans l'exemple $C$, les pensées de Menaud se rapprochent, selon un processus typiquement métonymique, de l'eau: eau du printemps, eau de la drave. Ailleurs, et plus souvent, c'est le feu qui sert de préférence à figurer le for intérieur du maître-draveur. Les deux exemples suivants sont à cet égard révélateurs: 
E. Au feu de cette lampe qui allumait des escarbilles en ses regards, il avait l'air d'un forgeron martelant des pensées de fer.

F. Aussi, son âme, ce matin, est-elle comme la cuve de forge quand le forgeron y plonge son fer rouge.

De telles images rassemblent non seulement toute la gamme des significations élémentaires associées au feu (sacré, visionnaire, etc.) mais encore les différentes connotations du mot fer: main de fer, volonté de fer, siècle de fer. De plus, l'artisanat évoqué par le mot forgeron fait contraste avec l'impersonnel et anonyme ingénieur de la compagnie (p. 24) qui, accompagné d'un interprète, vient demander, au nom de l'industrie et de l'argent anglais, les services du «seigneur et maître» de la rivière ${ }^{21}$.

C'est probablement la métaphore de la forge et des pensées de fer qui engendre ou suggère, encore une fois selon une logique métonymique, cette nouvelle figure des mots en armes que l'on trouve un peu plus loin à la même page:

G. Mais voilà que, de cette diable de lecture, étaient sortis des mots en armes, et qu'avec eux toutes les voix du pays, de la montagne, des champs et des bois s'étaient engouffrées chez lui, ébranlant son âme et sa maison comme une bourrasque d'automne.

L'image des voix du pays, héritée de Maria Chapdelaine, reviendra à maintes reprises tout au long du livre. Remarquons qu'elle s'assimile déjà ici au vent, à une bourrasque d'automne qui menace l'espace protecteur de la maison et sème le trouble dans l'âme du protagoniste. Ce sont ces voix, ces mots en armes, qui provoquent l'état d'excitation mentale que nous avons déjà constatée chez Menaud et qui le poussent sans cesse à partir, à renoncer aux conforts de la maison, de la vie sédentaire des femmes et des paysans:

H. Sa femme avait tout fait pour enraciner au sol ce fier coureur de bois. Et lui, par amour pour elle, il avait défriché cette âpre terre de Mainsal, toujours prêt, cependant, à s'évader du regard vers le bleu des monts dès que le vent du Nord venait lui verser au coeur les paroles magiques et les philtres embaumés.

(p. 23).

Le rapprochement vent $d u$ Nord/voix du pays revient plus loin dans une image caractéristique: 
I. Tout y avait passé dans le pays, et depuis le commencement; et cette parole rude et forte avait fouaille deux heures de temps dans la fumée des pipes, comme le noroît quand il fouette les nuages et découvre la vraie face de la terre.

La comparaison, loin d'être simple, est construite selon une formule chère à Savard, celle de l'homologie à quatre termes:

parole : noroît : : fumée des pipes: nuages

La même structure se retrouve dans cette autre image, où l'analogie entre la parole et le feu est encore plus explicite:

J. Voilà maintenant que cette parole flambait dans l'humble maison comme un feu d'abatis dans la clairière du printemps.

(parole: feu d'abatis : : humble maison: clairière du printemps)

On en trouve à la même page une variante un peu plus complexe:

K. Puis, il ouvrit la porte toute grande; et, dans le soir immobile, il contempla la campagne endormie, laissant ses regards voler jusqu'aux horizons lointains, et revenir ainsi que des engoulevents au nid de ses pensées.

La symétrie qui devrait s'établir entre les termes de l'homologie (regards: engoulevents : : horizons lointains: nid de ses pensées) se voit ici comme déséquilibrée par l'introduction de deux verbes (voler, revenir) au lieu d'un seul. On y retrouve la dialectique du dehors et du dedans, de l'ouvert et du fermé (horizons/nid) ainsi que celle du départ et du retour (voler/revenir) que nous avons vues dans les exemples A, B et $\mathrm{H}$. Cependant, l'articulation de ces deux dialectiques est désaxée par le fait que les termes de la comparaison (regards et engoulevents) sont les sujets de deux verbes à sens unique et opposé: ce sont les regards qui volent et les engoulevents qui reviennent. L'instabilité de l'image s'explique peut-être en partie par l'ambiguïté de la symbolique de l'oiseau dans l'œuvre, les engoulevents participant à la fois de l'oiselet que le vent bourru déniche (p. 199) et de l'outarde qui regagne le Nord avec du vent neuf plein ses ailes (p. 181). De même, la dialectique du dehors et du dedans se voit compliquée par le fait que la campagne endormie - qui renvoie par métonymie à l'image de la grande paix dorée du soir s'étendant comme une couverture qu'une femme étale sur un lit (p. 19) s'oppose, non pas à l'univers fermé de la maison, mais plutôt à celui des horizons lointains, de la montagne et des bois. André Brochu s'est attaché à cette distinction dans son article "Menaud ou l'impossible fête": Tout le roman, écrit-il, montrera d'ailleurs que la maison et la nature qui se dispose autour - la nature des champs et non celle des montagnes - sont intimement liées et composent ensemble l'univers dévolu à la femme [...] les champs sont le prolongement de la maison, qui est elle-même le prolongement de la chambre $^{22}$. 
Cependant, même la femme, dans la sécurité de sa chambre, n'est pas à l'abri de cette autre nature, la nature sauvage dont le porte-parole est la bourrasque, le vent qui est l'âme même de la montagne:

L. Ensuite, elle regagna sa chambre, saisie par cette bourrasque froide qui l'avait frappée au visage comme la première annonce des peines qui l'attendaient.

L'image reviendra avec insistance quelques pages plus loin, dotée cette fois de son plein sens métaphorique, chargée de l'âpre morale des ancêtres, mais d'une morale curieusement domestiquée, apprivoisée, selon le point de vue borné de Marie:

M. A tout instant la belle heurtait du regard contre les souvenirs de sa mère pieusement disposés ça et là.

Alors elle s'assit, toute figée, tandis que la bourrasque secouait la vieille maison, et que la poudrerie grêlait les vitres...

Ça me reproche! pensa-t-elle... ça me reproche!

Le reproche communiqué par la bourrasque se fera entendre une fois de plus dans ce chapitre, mais il sera transformé dans le cœur du Lucon qu'il disputera avec cette «incantation d'amour» qu'est la parole de Marie:

N. Mais les paroles de Marie sonnaient encore, sonnaient autour de lui comme les grelots d'une carriole de noces:

"Ce serait plaisant de vivre icitte... tranquille!"

Avant de prendre la dépente de la côte, il s'arrêta pour regarder la lumière de la maison promise; mais une bourrasque venue de la montagne lui jeta au visage un air froid, comme un reproche.

On revient ainsi à la même dialectique que nous avons constatée dans nos premiers exemples (A et B), transposée ici en termes du dilemme d'Alexis: rester ou partir, se marier et s'enraciner au sol ou bien répondre à l'appel du pays et de la race, écouter les paroles magiques, les mots en armes qui l'incitent à défendre le patrimoine contre les étrangers.

Dans les exemples que nous avons étudiés, nous avons constaté la part dévolue à la métonymie dans le procès métaphorique. Dans tous les cas, c'est la métonymie qui, selon le mot de Genette, authentifie le rapport d'analogie en l'intégrant à la diégèse, au système de relations - tantôt spatiotemporelles, tantôt psychologiques - qui constitue l'univers romanesque. Quoi de plus naturel, par exemple, de plus authentique, que cette vision d'un peuple fort comme le printemps lorsque le soleil descend sur le pays et donne des coups de pique sur les embâcles de l'hiver (p. 32)? Ou encore, pour évoquer l'état d'âme de Marie, après l'arrivée du Délié, le prétendant: Mais le 
dialogue silencieux qu'échangeaient son cour et la pensée de son père, ainsi qu'une laine fragile, était rompu (p. 27). Faut-il rêver sur la signification du nom Délié avec sa suggestion de liens rompus? Ou serait-ce plutôt la faiblesse des femmes, leur trahison éventuelle, qui est sous-entendue par cette laine fragile?

Non, Menaud n'aimait point ce gars-là! De tout son instinct d'homme libre et jaloux du sol. Pour lui, le Délié était un de ces traitres, un de ces vendus qui livrent, pour de l'argent, la montagne et les chemins à l'étranger.

Cette espèce-là, sans doute, quand on en aurait assez... tout le pays, toute la race la renieraient. épouser...

De penser que sa fille, le sang de son sang, pourrait un jour...

Cela, non! Jamais!

De toute façon, l'authenticité de l'image est comme surdéterminée, tant par ce fond de spéculation thématique que par le fait que Marie elle-même est assise à son métier et vient de reprendre son travail de tisserande.

Remarquons pourtant que ce travail est lui-même doté d'un sens métaphorique:

Et l'on n'entendait plus que le frappement du ros qui tassait la tissure entre les fils de la chaine.

Un rythme la berçait de droite à gauche. De ses deux bras harmonieusement levés l'un après l'autre, elle semblait battre la mesure à quelque mystérieuse musique, cependant qu'à la trame de cette lourde étoffe grise, elle insérait toute la chaleur de son être pour son père, pour Joson, qu'elle protégerait ainsi contre le froid qui glace là-bas le cour des hommes.

Et c'était sa manière à elle de dire à chaque coup de marchette: "Une race qui ne sait pas mourir!»

Ce qu'elle faisait là, sa mère et bien d'autres femmes l'avaient fait avant elle, entremélant aux laines de subtils sentiments de force, de résistance, et des prières même.

C'est ainsi que l'image de la laine fragile - par un processus à la fois métonymique et métaphorique (laine - tisserande - fidélité au passé) - rejoint celle, en apparence toute différente, du soleil transformé en maître-draveur:

Et ce fut alors comme s'il l'eût vue, cette race, non dans les livres, mais vivante, mais dans sa chair dressée là, devant lui; et cette race, elle devenait comme un grand peuple libre, debout, enfin, dans sa lumière, et fort comme le printemps lorsque le soleil descend sur le pays et donne des coups de pique sur les embâcles de l'hiver. 
C'est, en dernière analyse, cette vision de la race - et l'idéologie de la survivance et de la fidélité qu'elle engendre - qui informe, à travers un réseau d'oppositions thématiques, toutes les images que nous avons relevées. Mais si la cohérence de l'œuvre réside avant tout dans le rapport métaphorique qui unit l'univers romanesque à un monde métaphysique de mythes et de visions héroïques, sa cohésion relève plutôt de la logique métonymique qui soude les rapports - horizontaux plutôt que verticaux - s'établissant entre les différents éléments de la diégèse.

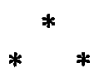

Nous nous sommes borné jusqu'ici à une étude du jeu de la métaphore et de la métonymie au seul niveau des figures et, plus particulièrement, du rôle de la métonymie dans la métaphore. Rappelons pourtant que l'opposition entre métaphore et métonymie est susceptible d'être généralisée pour rejoindre celle, fondamentale, entre les deux axes du langage, celui de la sélection et celui de la combinaison, axe paradigmatique et axe syntagmatique. Comme le dit Roman Jakobson dans son étude classique de l'aphasie, c'est par sa manipulation de ces deux aspects du langage que l'individu révèle son style personnel, ses goûts et préférences verbales:

Le développement d'un discours peut se faire le long de deux lignes sémantiques différentes: un thème (topic) en amène un autre soit par similarité soit par contiguité. Le mieux serait sans doute de parler de procès métaphorique dans le premier cas et de procès métonymique dans le second, puisqu'ils trouvent leur expression la plus condensée, l'un dans la métaphore, l'autre dans la métonymie ${ }^{23}$.

D'après Jakobson, les troubles aphasiques peuvent être divisés en deux catégories selon qu'ils affectent la faculté de sélection ou celle de combinaison. Dans le premier type c'est la relation de similarité qui est altérée, dans le second celle de contiguitté. Pour un malade souffrant du trouble de la similarité, le contexte (linguistique et référentiel) devient un facteur indispensable et décisif. Un tel malade complète des fragments avec beaucoup de facilité, son discours n'étant fait que de réactions, mais il trouve difficile d'amorcer un dialogue et encore plus difficile d'exécuter ou même de comprendre un discours clos comme un monologue ${ }^{24}$. Il a du mal à nommer un objet ou à définir un mot. Au lieu de dire 'ceci est [appelé] un crayon' il ajoutera simplement une remarque elliptique concernant son usage: 'Pour écrire'25. Il trouve même difficile de répéter un mot prononcé par un autre, toute répétition lui semblant inutilement redondante. Le sujet de la phrase est souvent omis et les mots-clés sautés ou remplacés par des substituts anaphoriques abstraits, tandis que les mots qui comportent une référence inhérente au contexte, tels que les pronoms et les adverbes pronominaux, et les mots servant à construire le contexte tels que les connectifs et les auxiliaires sont particulièrement aptes à survivre ${ }^{26}$. C'est donc la charpente du discours, les chaînons de connexion de la communication ${ }^{27}$ qui ont tendance à rester 
intacts dans ce type d'aphasie. Dans le trouble de la contiguïté, par contre, on constate une altération de l'aptitude à construire des propositions, ou, en termes plus généraux, à combiner des entités linguistiques simples en unités plus complexes... ${ }^{28}$ :

Dans ce type d'aphasie, déficiente quant au contexte, et qu'on pourrait appeler trouble de la contiguïté, l'étendue et la variété des phrases diminuent. Les règles syntaxiques qui organisent les mots en unités plus hautes sont perdues; cette perte, appelée agrammatisme, aboutit à dégrader la phrase en un simple 'tas de mots', pour nous servir de l'image de Jackson. L'ordre des mots devient chaotique; les liens de coordination et de subordination grammaticales, soit d'accord ou de rection, sont dissous. Comme on pouvait s'y attendre, les mots dotés de fonctions purement grammaticales, tels que les conjonctions, prépositions, pronoms et articles, disparaissent en premier lieu pour faire place au style dit "télégraphique», alors que dans le cas du trouble de la similarité ils sont les plus résistants. Moins un mot dépend grammaticalement du contexte, plus forte est sa persistance dans le discours des aphasiques chez qui la fonction de contiguité est atteinte et plus tôt il est éliminé par les malades souffrant d'un trouble de la similarité. Ainsi le sujet, "mot noyau» est-il le premier à disparaître de la phrase dans les cas de trouble de la similarité et, par contre, le moins destructible dans le type opposé d'aphasie. L'aphasie dans laquelle la fonction du contexte est affectée tend à ramener le discours à d'infantiles énoncés d'une phrase, voire à des phrases d'un mot. Seules quelques phrases plus longues, mais alors stéréotypées, "toutes faites», parviennent à survivre ${ }^{29}$.

La question se pose alors de savoir si l'on peut reconnaître, dans l'une ou l'autre de ces deux tendances générales, le style personnel de F.-A. Savard, ses goûts et préférences verbales. Pour nous mettre à l'abri de toute accusation d'avoir faussé les termes de l'analyse, contentons-nous de reproduire ici les conclusions de l'excellente étude de F. Ricard. Le chapitre que le critique consacre aux liaisons est à cet égard lumineux. Ricard commence par constater l'extrême abondance des énumérations qui, s'effectuant sous le signe de l'accumulation, visent à amplifier les descriptions tout en intensifiant l'émotion ${ }^{30}$. Cependant, une telle technique n'est pas sans danger et, comme le remarque Ricard, à relire Menaud d'une façon plus attentive, on ne tarde pas à découvrir le secret, qui parfois confine à la recette. Une pointe de déception vient alors, et par endroits, l'éblouissement de jadis se change en une vague lassitude, devant la facilité de certaines surcharges ${ }^{31}$. De même, on a parfois l'impression que les mots sont superflus, que l'emploi de plusieurs termes parents ne fait que trahir l'absence du mot unique qui les résumerait tous et que l'auteur n'a pas su découvrir ${ }^{32}$. Ce qui, par contre, donne à ce procédé son expressivité, c'est avant tout l'absence de coordination, à laquelle l'auteur préfère la simple juxtaposition. Il en va de même en ce qui concerne les liaisons entre les propositions: Dans Menaud, les indépendantes fourmillent. Parfois coordonnées par un et de relance, un peu comme chez Flaubert, elles 
sont le plus souvent simplement juxtaposées ${ }^{33}$. Quant à la phrase complexe, elle est reléguée aux oubliettes dans le style analytique de Savard ${ }^{34}$, la subordination se réduisant presque exclusivement aux relatives et aux comparatives, car l'abbé Savard préfère les liaisons faibles, tout juste suffisantes à faire sentir un rapport indéterminé entre les propositions ${ }^{35}$. Le même souci se manifeste encore au niveau des liaisons entre les phrases: Rares sont, dans Menaud, maître-draveur, les ensembles élaborés, dont les phrases, participant à un tout cohérent, développeraient à la suite les uns des autres différents aspects d'une idée centrale. Au contraire, Savard affectionne la phrase détachée, autonome, formant le plus souvent paragraphe ${ }^{36}$. C'est ainsi que se forme ce que Ricard appelle un style pointilliste, Savard disposant ses paragraphes comme autant de détails particuliers sans les relier grammaticalement d'une façon étroite. Cela ne va pas, toutefois, sans le risque d'une fragmentation excessive. $\dot{A}$ la longue, passant d'un paragraphe à l'autre, le lecteur se demande où l'on va ${ }^{37}$.

Si donc notre auteur pèche par excès, il est clair que cet excès relève de la métaphore plutôt que de la métonymie. Sans vouloir parler d'un trouble de la contiguité dans le cas de Savard, il nous semble indéniable que le style maniéré de Menaud, maître-draveur se rapproche nettement du pôle métaphorique du langage, privilégiant les rapports paradigmatiques de similarité aux dépens de la continuité et de la coordination syntagmatiques. Style vertical plutôt qu'horizontal, fait d'extases poétiques et de morceaux d'anthologie, il risque sans cesse de tarir le maigre filet du récit et de mettre fin à toute progression narrative.

Il est intéressant de remarquer que le critique Yvon Malette se sert dé la même opposition entre vertical et horizontal pour distinguer le roman de Savard de Maria Chapdelaine:

Hémon nous fait participer à l'expérience romanesque de ses personnages mais Maria Chapdelaine nous apparaît d'abord comme une peinture réaliste de ces bonnes gens, d'où l'emploi d'un style d'information et l'impression alors d'une action située sur un plan plutôt horizontal. On ne retrouve pas un tel tableau dans le roman de Savard car la folie du draveur est un avertissement préparé graduellement, d'où l'impression d'une "action-escalier", d'une action située sur un plan plutôt vertical ${ }^{38}$.

Là où Louis Hémon adopte un style à la fois descriptif, narratif et réaliste, Savard adopterait, de son côté, un style à la fois lyrique, poétique et épique ${ }^{39}$. Chose curieuse, on rejoint par là un autre aspect de notre distinction fondamentale entre métaphore et métonymie, car Jakobson voit dans la prédominance de l'une ou de l'autre dans un texte un moyen de caractériser le genre littéraire auquel le texte appartient:

La primauté du procédé métaphorique dans les écoles romantiques et symbolistes a été maintes fois soulignée mais on n'a pas encore suffisamment compris que c'est la prédominance de la métonymie qui gouverne et définit effectivement le courant litté- 
raire qu'on appelle 'réaliste', qui appartient à une période intermédiaire entre le déclin du romantisme et la naissance du symbolisme et qui s'oppose à l'un comme à l'autre. Suivant la voie des relations de contiguïté, l'auteur réaliste opère des digressions métonymiques de l'intrigue à l'atmosphère et des personnages au cadre spatio-temporel ${ }^{40}$.

Nous revenons ainsi à notre point de départ, à la constatation d'un malaise de la part des critiques devant l'apparente dualité de l'ouvrage de l'abbé Savard. Il convient maintenant d'approfondir cette question en confrontant les structures stylistiques et les structures thématiques de l'œuvre.

Toute lecture thématique de Menaud, maître-draveur doit commencer par les extraits de Maria Chapdelaine, véritable clef de voûte de l'édifice romanesque. Or, dans un article convaincant, François Martineau a constaté que la fonction de ces extraits dans le roman de Savard implique une transformation de leur sens originel et donc de la structure thématique de l'œuvre entière. S'attachant surtout à la phrase Autour de nous des étrangers sont venus, il s'efforce de montrer que, pour Hémon, autour de nous ne signifie pas ici, au Québec. Chez Savard, par contre, les étrangers sont bien ici, non autour de nous mais parmi nous, ce qui se reflète dans le fait que les mots Des étrangers sont venus reviennent à douze reprises dans le texte, tandis que le membre de phrase Autour de nous n'apparaît qu'une seule fois. L'une des conséquences de ce changement historique se manifeste dans le déplacement thématique du dilemme de Maria/Marie:

On peut avancer que Maria, si elle cédait à la tentation américaine incarnée dans Lorenzo Surprenant, ne créerait pas cependant un trop grand problème. Elle partirait à l'extérieur de son pays laissant les siens entre eux. C'est le problème de l'émigration. Mais Savard, lui, veut montrer le problème de la présence de l'étranger ici. Une des conséquences, c'est le morcellement, la scission à l'intérieur même de la collectivitét ${ }^{4}$.

De Maria Chapdelaine à Menaud, maître-draveur, le jeu des oppositions a été désaxé, son sens renversé. La dialectique de l'ici et de l'ailleurs n'a plus la même valeur. Chez Hémon, le simple fait de rester constituait une affirmation de continuité avec le passé; partir, c'était partir pour les États-Unis et donc trahir l'héritage. Chez Savard, par contre, le verbe partir a toujours un sens positif, associé comme il l'est au mode de vie des coureurs de bois, des nomades; partir, c'est partir pour la montagne, cet espace mythique qui s'oppose aux champs et aux maisons des sédentaires. Dans Menaud, partir c'est donc affirmer sa fidélité aux valeurs des ancêtres; rester c'est nécessairement trahir ces mêmes valeurs. Comme le dit Martineau; Savard a transposé dans son Menaud son inquiétude vis-à-vis le sort de sa collectivité menacée 
de désintégration. Pour ce, il ne pouvait faire mieux que de se référer à cet ouvrage qui cristallisait une certitude d'alors (1914) maintenant écroulée $(1937)^{42}$.

Cependant, il serait faux de parler de deux collectivités - de sédentaires et de nomades - dans le roman de Savard, car tout le drame de Menaud réside dans l'échec du protagoniste, son impuissance à rallier une communauté de fidèles contre l'étranger. C'est sans doute pourquoi André Brochu préfère situer l'opposition sur un autre plan en distinguant, d'une part, une collectivité dans l'espace - celle des hommes du présent - et, d'autre part, une collectivité dans le temps - celle des ancêtres, de la lignée, celle des 'héros de mon pays'43. Or, nous nous sommes déjà servi d'une distinction stylistique et structurale entre les dimensions verticale et horizontale de l'œuvre, distinction qui reçoit dans l'étude de Brochu une formulation purement thématique:

Résumons-nous: il existe, dans Menaud, une relation certaine entre la nature, et plus exactement l'étendue horizontale (qui s'oppose à la nature verticale de la montagne), la femme et l'étranger d'une part; entre la pensée (fidélité au passé, à la profondeur temporelle), l'homme (Menaud, Joson, le Lucon) et les ancêtres d'autre part. On verra aussi que c'est dans la montagne que Menaud entre le plus intimement en contact avec ses pères: la hauteur dans l'espace est liée à la profondeur dans le temps, et toutes deux impliquent un même refus de l'étendue, c'est-à-dire de l'étranger et, plus secrètement, de la femme ${ }^{44}$.

La question se pose alors de savoir s'il existe une corrélation entre le sens formel et le sens thématique de l'opposition horizontal/vertical. Rappelons que sur le plan stylistique l'opposition entre vertical et horizontal reprend celle entre métaphore et métonymie. Le style métonymique (ou horizontal) est à la fois descriptif, narratif et réaliste, orienté vers la diégèse, tandis que le style vertical (ou métaphorique) est à la fois lyrique, poétique et épique. Or, dans un article rédigé pour le Dictionnaire des œuvres littéraires du Québec, François Ricard s'est efforcé de caractériser ce qu'il appelle la dimension lyrique du roman - faite de métaphores, de visions et des voix de Maria Chapdelaine - de la manière suivante:

Celle-ci, formée de l'ensemble des symboles, des souvenirs ou des mythes qui peuplent le récit, est comme un vaste univers imaginaire se superposant à l'univers immédiat de choses et d'événements dans lequel évoluent les personnages. Constamment, le lecteur est amené à passer de l'aventure de ces derniers, du décor particulier de Charlevoix ou du temps réel dans lequel se déroulent les événements, à une Aventure, un Décor et un Temps proprement idéaux ou surhumains qui les doublent, pour ainsi dire, et leur servent d'arrière-fond héroïque ${ }^{45}$. 
C'est-à-dire que la distinction entre style vertical et style horizontal oppose, d'une part, l'ensemble des symboles, des souvenirs ou des mythes et, d'autre part, l'univers immédiat de choses et d'événements dans lequel évoluent les personnages. Or, nous voici très proche de la distinction thématique faite par André Brochu entre la collectivité dans le temps (celle de la lignée) et la collectivité dans l'espace (celle des personnages). Quant à nous, nous préférons parler d'un espace-temps métaphorique, fait de rapports in absentia et orienté vers un passé mythique, et d'un espace-temps métonymique, fait de rapports in praesentia et orienté vers le présent de la diégèse.

C'est à cette tension entre deux styles différents que Ricard attribue l'embarras des critiques devant le problème du genre littéraire dans lequel il convient de ranger Menaud:

Cette ambiguité, on l'aura compris, provient surtout du style assez particulier de l'ouuvre, qui mêle sans cesse le narratif au lyrique et qui, tout en offrant l'aspect général d'un roman, avec intrigue, dialogues et personnages, utilise cependant des procédés généralement réservés à la poésie: abondance des métaphores, usage de l'apostrophe héroïque et de la prosopopée, récherche rythmique... ${ }^{46}$

Hâtons pourtant d'ajouter que cette explication "stylistique» se doit d'être nuancée, car, comme nous l'avons vu, sur le plan des figures elles-mêmes, la métonymie joue un rôle important et contribue à ancrer les métaphores et les comparaisons dans la diégèse. Il s'ensuit que les sources de cette ambiguité ou de ce malaise sont à chercher ailleurs que dans le style au sens restreint du terme, à un niveau plus profond. C'est André Brochu qui nous indique le chemin qui est à suivre, lorsqu'il écrit que Menaud n'est pas, comme certains l'ont soutenu, une oeuvre épique. Dans l'épopée, les 'méchants' se présentent concrètement devant le héros et sont affrontés d'égal à égal. Ici, les étrangers demeurent une forme anonyme... ${ }^{77}$ Or, cette épopée, il nous semble que l'abbé Savard aurait bien voulu l'écrire, qu'il aurait bien voulu chanter le triomphe de l'individu sur une collectivité devenue anonyme et impersonnelle. Cependant, l'auteur s'est heurté à un obstacle, 'et cet obstacle c'était la réalité de la situation historique. Impossible alors de raconter l'histoire héroïque du démiurge Menaud quand l'homme qu'il cache est écrasé par l'Histoire: $I l$ s'agit, écrit Ricard, pour le vieux maître-draveur de plier le présent historique à sa vision a-temporelle, de rendre le réel ambiant conforme à un certain langage dépositaire de l'idéal ${ }^{48}$. C'est l'impuissance de Menaud à réaliser ce projet qui finira par le plonger dans la folie.

L'affrontement, l'épreuve de force tant voulue par Menaud, ne se produit donc pas. Les termes du combat ne sont plus les mêmes. Menaud vit toujours dans le monde des gestes héroïques, le monde du western où un individu exceptionnel est encore capable de sauver la collectivité. Cependant, le monde a changé: l'oppression et l'exploitation sont devenues anonymes, n'ont plus de visage particulier, reconnaissable, mais se cachent derrière la compagnie, l'économie, la loi. Le Délié, lui, n'est qu'un symbole vide, un homme de 
paille, un bouc émissaire. Et quant à l'étranger, il n'est remarquable que par son absence; il se réfugie à Wall Street ou à Bay Street, à la Bourse et dans les salles du conseil des grandes compagnies. C'est ce que Menaud ne comprend point, parce que Menaud est un anachronisme. Sa collectivité à lui, c'est la tribu et non la société moderne. Rendu dans la montagne pour se battre. pour défendre l'héritage contre un ennemi éternellement absent, le guerrier se transforme du jour au lendemain en vieillard pathétique qui vivra désormais au passé du conditionnel, au temps de l'impuissance et de l'échec, au temps, à la limite, de la folie.

Récapitulons. Sur le plan des images, la métonymie exerce une fonction d'authentification et de naturalisation, intégrant les métaphores à la diégèse. Pourtant, celle-ci est elle-même déjà si métaphorique - le cycle des saisons, la valeur thématique et idéologique de l'espace: le pays, les bois, la montagne, les champs, la maison, la cambuse, etc. - qu'il n'y a aucun doute quant au mode dominant: la métonymie joue un rôle important mais secondaire. Sur le plan du style au sens plus large du terme, la prédilection de Savard pour la métaphore et pour l'aspect vertical de l'écriture se manifeste dans son insistance sur l'importance de lutter contre le temps en inscrivant des choses au-dessus de son cours ${ }^{49}$. Une telle recherche esthétique de l'absolu privilégie de toute évidence le langage poétique aux dépens d'un réalisme romanesque ancrè dans le déroulement métonymique du récit. Ce qui ne poserait pas trop de problèmes si les thèmes traités s'accordaient à une telle élévation du style, laquelle exigerait une célébration épique d'exploits héroïques accomplis au nom d'une idéologie unifiée et sans faille. Cependant, il devient clair au cours du récit que la société moderne ne peut plus nourrir une telle vision du monde ni soutenir un tel style. Le monde refuse de collaborer à ce genre de transformation métaphorique du réel. C'est cette fracture de l'illusion métaphorique qui conduit à la folie de Menaud, folie tout aussi littéraire que celle de Don Quichotte ${ }^{50}$. Et là encore, même la folie de Menaud s'avère métaphorique: C'est pas une folie comme une autre! dit Josime. Ça me dit, à moi, que c'est un avertissement (p. 205). C'est une folie qui signifie, qui renvoie à autre chose. L'écriture ne permet jamais à l'univers romanesque de se contenter d'exister selon quelque illusion réaliste ou métonymique, mais préfère, stylistiquement, se réfugier dans les oracles sybillins de la folie pour figurer l'échec de la cohérence métaphorique.

Menaud, comme le dit François Ricard, est un héros épique jeté dans un monde romanesque. En d'autres mots, un être dominé par une pensée profondément mythique, qui se voit plongé dans un univers négateur, où ses plus chères aspirations se brisent contre l'obstacle de la désolante réalités1. Et ce trajet de Menaud, c'est le trajet d'un genre à l'autre, de l'épopée au roman, de la métaphore à la métonymie; trajet pénible et accompli sans doute à contrecœur, mais qui représente, par son incohérence même, un premier pas vers le roman québécois moderne. Il est difficile à un ouvrage, écrit Madeleine Ducrocq-Poirier, de vivre une intériorité déviée de son projet initial ${ }^{52}$. Ajoutons pourtant que c'est précisément dans cette difficulté d'être que réside tout l'intérêt, à la fois historique et littéraire, du roman de Savard. 
1. Maurice Hébert, "Menaud, maître-draveur'," le Canada français, vol. XXV, no 3, novembre 1937, p. 335.

2. Madeleine Ducrocq-Poirier, le Roman canadien de langue française de 1860 à 1958. Recherche d'un esprit romanesque, Paris, Nizet, 1978, p. 332.

3. Ibid., p. 333.

4. Réjean Robidoux et André Renaud, le Roman canadien-français du XXe siècle, Éditions de l'Université d'Ottawa, 1966, p. 41.

5. Ibid., p. 43.

6. François Ricard, l'Art de Félix-Antoine Savard dans «Menaud, maître-draveur», Montréal, Fides, 1972, p. 39-40. On retrouve la même conclusion dans l'article de Louise Vanhee-Nelson, "Trois versions de 'Menaud, maître-draveur'», Essays on Canadian Writing, 15, été 1979, p. 94: Le texte plus continu de la deuxième version donne une impression de récit plus touffu, plus lié à une réalité parfois ambiguë. Reste à déterminer la nature et la source de cette ambiguïté. Sur la question des différentes éditions, on consultera aussi l'étude récente de Jules Tessier, "Le mythe des trois versions de 'Menaud, maître-draveur'", dans la Revue d'histoire littéraire du Québec et du Canada français, no 4, été-automne 1982, p. 83-90. L'auteur nous rappelle qu'il n'y a pas trois versions de Menaud, mais cinq: celles de 1937, 1938, 1944, 1960 et 1964. Il nous signale aussi qu'il existe des différences importantes entre l'édition de 1937 et celle, définitive, de 1964.

7. Ricard, p. 29-30.

8. Ibid., p. 125.

9. Félix-Antoine Savard, Menaud, maître-draveur, présentation d'André Renaud. chronologie, bibliographie et jugements critiques d'Aurélien Boivin, Montréal, Fides, 1982, p. 156.

10. Ibid., p. 166. Désormais les références à cette édition seront données entre parenthèses dans le texte de l'article.

11. Gérard Genette, «Métonymie chez Proust,», dans Figures III, Paris, Éditions du Seuil, 1972, p. $47-48$.

12. Ibid., p. 41 .

13. Stephen Ullmann, Style in the French Novel, Cambridge University Press, 1957. Cité par Genette, p. 41.

14. Ullmann, p. 197. Cité par Genette, p. 41.

15. Genette, p. 42.

16. Ibid., p. 43.

17. Ibid.

18. Ibid., p. 43-44.

19. Ibid., p. 45. Il ajoute en note: En fait, la motivation est réciproque et joue dans les deux sens: la proximité authentifie la ressemblance, qui autrement pourrait sembler gratuite ou forcée, mais en retour la ressemblance justifie la proximité, qui autrement pourrait sembler fortuite ou arbitraire, sauf à supposer (ce qui n'est pas) que Proust décrit tout simplement un paysage qu'il a 'sous les yeux'.

20. Ibid.

21. La qualité d'artisan de Menaud est mise en évidence (et valorisée) dans l'image suivante qui rapproche encore une fois la continuité avec le passé et cette même excitation intellectuelle qu'on a déjà constatée dans d'autres images: Patiemment, suivant le rite hérité des ancêtres, il lace les nerfs de ses raquettes. Ainsi apaise-t-il sa tête peuplée d'idées en marche par des gestes d'artisan qui signifient volonté de conquête et passion de libre espace (p. 154).

22. André Brochu, "Menaud ou l'impossible fête" dans l'Instance critique, Montréal, Leméac, 1974, p. 257-58. 
23. Roman Jakobson, Essais de linguistique générale, Paris, Éditions de Minuit, 1963, p. 61.

24. Ibid., p. 50 .

25. Ibid., p. 52.

26. Ibid., p. 51 .

27. Ibid.

28. Ibid., p. 57.

29. Ibid., p. 57-58.

30. F. Ricard, op. cit., p. 80.

31. Ibid., p. 80-81.

32. Ibid., p. 81.

33. Ibid., p. 82-83.

34. Ibid., p. 83.

35. Ibid., p. 85 .

36. Ibid.

37. Ibid., p. 86 .

38. Yvon Malette, “'Menaud, maître-draveur' et 'Maria Chapdelaine',"Incidences, no 13, hiver 1968 , p. $23-24$

39. Ibid., p. 21 .

40. R. Jakobson, op. cit., p. 62-63.

41. François Martineau, "Le sens originel des extraits de 'Maria Chapdelaine' et leur interprétation dans Menaud, maître-draveur'," Co-Incidences, Vol. II, no 3, novembre 1972, p. 17-18.

42. Ibid., p. 19.

43. André Brochu, op. cit., p. 254.

44. Ibid., p. 264.

45. François Ricard, “'Menaud, maître-draveur', roman de l'abbé Félix-Antoine Savard,» dans Dictionnaire des oeuvres littéraires du Québec. tome II, 1900-1939, Montréal, Fides, 1980, p. 695.

46. Ibid., p. 696.

47. Brochu, p. 253.

48. Ricard, l'Art de Félix-Antoine Savard dans «Menaud, maître-draveur», p. 113.

49. Cité par André Major, Félix-Antoine Savard, Montréal, Fides, 1968, p. 11-12.

50. Cf. F. Ricard, l'Art de Félix-Antoine Savard, p. 114: Il n'est pas absurde de croire que l'égarement du mấtre-draveur, en effet, est d'ordre purement verbal, ce qui en accentue d'autant la portée tragique: Menaud devient étranger à lui-même à cause d'un langage qui n'est pas de lui. On trouve la même idée dans Jean Ethier-Blais, «Félix-Antoine Savard - Le vol des oies sauvages,» dans Signets II, Ottawa, Le Cercle du Livre de France, 1967, p. 137. Cf. aussi Louise Vanhee-Nelson, art. cit., p. 96.

51. Ricard, p. 117.

52. M. Ducrocq-Poirier, op. cit., p. 333.

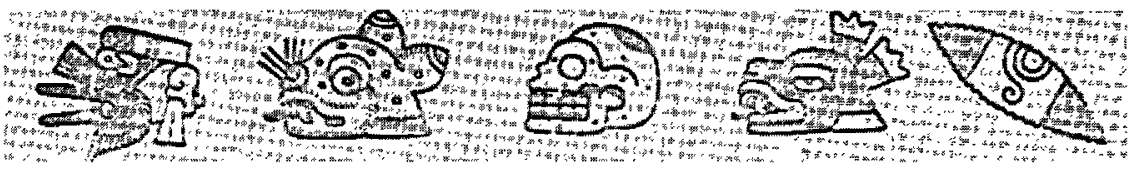




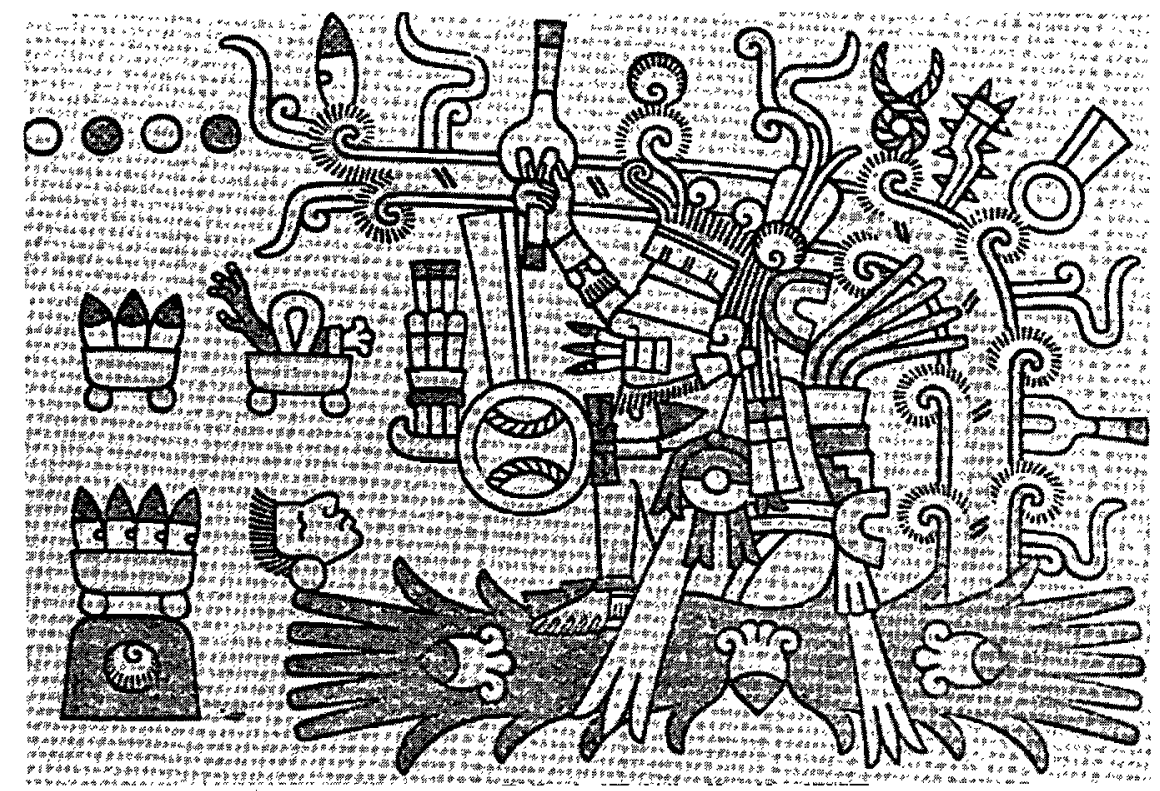

